\title{
Utility of Routine Left Ventricular Ejection Fraction Measurement Before Anthracycline-Based Chemotherapy in Patients With Diffuse Large B-Cell Lymphoma
}

\author{
By Amber L. Conrad, PA-C, Jacob D. Gundrum, MS, Vicki L. McHugh, MS, and Ronald S. Go, MD, FACP \\ Gundersen Lutheran Health System; and Gundersen Lutheran Medical Foundation, La Crosse, WI
}

\begin{abstract}
Purpose: Despite the lack of evidence, routine left ventricular ejection fraction (LVEF) measurement in diffuse large B-cell lymphoma (DLBCL) before anthracycline-based chemotherapy (ABC) is recommended by practice guidelines and required in DLBCL trials in the United States.
\end{abstract}

Methods: We determined the frequency of the following in 197 consecutive patients with newly diagnosed DLBCL treated at our institution: one, LVEF measurement before ABC; two, finding of asymptomatic LV dysfunction (ALVD); and three, modification in treatment strategy as a result of LVEF measurement.

Results: The median age was 71 years, and $54 \%$ of patients were men. LVEF was measured in 128 patients (65\%) pretreatment, including in 15 with prior congestive heart failure (CHF).

\section{Introduction}

Anthracycline-based chemotherapy $(\mathrm{ABC})$ regimens are among the most effective regimens for patients with diffuse large B-cell lymphoma (DLBCL). Previously, cyclophosphamide, doxorubicin, vincristine, and prednisone (CHOP) was considered the standard of care in the United States. ${ }^{1}$ In the last decade, rituximab plus $\mathrm{CHOP}$ (R-CHOP) has proven to be superior to and has replaced CHOP. ${ }^{2-4}$ Patients with advanced-stage DLBCL generally receive six to eight $\mathrm{R}-\mathrm{CHOP}$ cycles, with maximum cumulative doxorubicin doses between 300 and $400 \mathrm{mg} / \mathrm{m}^{2}$. Data from randomized clinical trials showed that after 2 to 3 years of follow-up, grades 3 and 4 cardiac toxicities-including congestive heart failure (CHF) —were noted in approximately $2 \%$ of younger (age $<60$ years) and $8 \%$ of older patients. ${ }^{2,4}$

Before $\mathrm{ABC}$, cardiac left ventricular ejection fraction (LVEF) is now commonly measured in patients with DLBCL. This practice is recommended in the National Comprehensive Cancer Network (NCCN) clinical practice guidelines for nonHodgkin lymphoma, is endorsed by the American Heart Association and American College of Cardiology, and appears on Federal Drug Administration-approved labeling guidelines. ${ }^{5-7}$ It is also an eligibility requirement in most adult US cancer cooperative group clinical trials in which $\mathrm{ABC}$ is being investigated. However, subsequent serial measurements of LVEF during or after $\mathrm{ABC}$ are not recommended by the NCCN or by lymphoma trials. This baseline measurement of LVEF is being used as a screening tool to detect asymptomatic LV dysfunction
The reasons for not measuring LVEF were: clinically low risk for ALVD $(n=32)$, medical frailty $(n=15)$, palliative care $(n=3)$, ABC not standard therapy $(n=12)$, and prior $\mathrm{CHF}(\mathrm{n}=7)$. Among patients without prior CHF who had LVEF assessed $(n=$ 113), ALVD was detected in four (4\%), with LVEF ranging from $41 \%$ to $48 \%$. Four patients were not treated despite normal LVEF because of comorbidities and anticipated toxicities. In contrast, all four patients with ALVD received ABC. No patient had a modification in treatment strategy as a result of LVEF measurement. After a median follow-up of 60 months, among those who remained alive, CHF developed in $15 \%$ versus $6 \%$ of patients receiving $A B C$ who did and did not have LVEF measured, respectively $(P=.246)$.

Conclusion: Our findings challenge the utility of routine LVEF measurement in patients with DLBCL before ABC. Potential cost savings to our health care system could be substantial.

(ALVD), but the utility of this screening practice is not supported by evidence.

In 1979, Von Hoff et al ${ }^{8}$ retrospectively analyzed the National Cancer Institute database and identified total cumulative dose of anthracycline as a major risk factor for development of $\mathrm{CHF}$ after $\mathrm{ABC}$. Although the estimated overall incidence of clinical CHF after doxorubicin treatment was only $2.2 \%$, the incidence rose to $3 \%, 7 \%$, and $18 \%$ for patients who received cumulative doses of 400,550 , and $700 \mathrm{mg} / \mathrm{m}^{2}$, respectively. However, the study could have underestimated the true incidence of CHF, because many patients received relatively low doses of doxorubicin, and the reporting of CHF itself was based on investigator reporting. Subsequently, in 1987, Schwartz et $\mathrm{al}^{9}$ proposed a guideline of serial LVEF measurements according to normal or abnormal baseline LVEF to identify patients at risk of developing CHF. The guideline recommended that patients with $\mathrm{LVEF} \leq 30 \%$ not receive $\mathrm{ABC}$. For patients with LVEF between $30 \%$ and $50 \%$, they advised serial LVEF measurement and cessation of $\mathrm{ABC}$ in patients who had an absolute LVEF decline of $\geq 10 \%$. They found that patients who were treated according to the guideline were less likely to develop $\mathrm{CHF}$, or more likely to develop milder cases, than those who were not. A more recent study in 2003 by Swain et al ${ }^{10}$ evaluated data from three prospective randomized clinical trials that studied $\mathrm{ABC}$ with placebo versus dexrazoxane in patients with breast or lung cancer. Inclusion criteria included a normal institutional baseline LVEF (50\% for most institutions) as mea- 
sured by multiple-gated acquisition (MUGA) nuclear scans at study entry. LVEF was measured serially. ABC-induced clinical CHF (defined as $\geq$ two of the following: cardiomegaly on chest radiograph, basilar crackles, S3 gallop, and either paroxysmal nocturnal dyspnea, orthopnea, or significant dyspnea on exertion) was determined by a cardiologist blinded to the treatment arms. The estimated incidence of ABC-induced clinical CHF was markedly higher at higher cumulative doses: $5 \%, 16 \%$, and $48 \%$ among those receiving 400,550 , and $700 \mathrm{mg} / \mathrm{m}^{2}$, respectively. More importantly, LVEF was found to be an unreliable predictor of CHF, even if the guideline proposed by Schwartz et $\mathrm{al}^{9}$ was followed.

We retrospectively identified patients newly diagnosed with DLBCL at our institution over a 10 -year period to determine: one, frequency of LVEF measurement before $A B C$; two, frequency of finding ALVD; three, frequency of modification in initial treatment strategy as a result of LVEF measurement; and four, patterns of care among our hematologists and oncologists regarding $\mathrm{LVEF}$ measurement before $\mathrm{ABC}$.

\section{Methods}

All consecutive patients with DLBCL diagnosed and treated at our facility from January 2000 through September 2009 were identified using our Commission on Cancer-accredited Gundersen Lutheran cancer registry and were included in the study. The following data were collected: demographics, lymphoma information (stage, treatment, follow-up), LVEF measurement (if measured, the value and modality used; if not measured, the reason), number of CHF risk factors (male sex, obesity, hypertension, coronary artery disease [CAD], diabetes mellitus, current cigarette smoking, prior $\mathrm{ABC}$, prior chest radiotherapy), reasons for non- $\mathrm{ABC}$ treatment, and development of CHF after ABC. ${ }^{11}$ To determine the frequency of LVEF measurement before $\mathrm{ABC}$ among physicians, we compared the practice patterns of medical oncologists (as a group) with those of individual hematologists, because unless seen in rural outreach clinics, most patients with DLBCL in our cohort were treated by hematologists. A patient was considered lost to follow-up if no clinical update was obtainable within 1 year of November 1, 2011. We used the Fisher's exact or $\chi^{2}$ test to compare study groups for categorical variables and the Wilcoxon rank sum test for comparison of the number of $\mathrm{CHF}$ risk factors. A $P$ value of less than .05 was considered statistically significant.

\section{Results}

We identified 197 patients who met study criteria. The median age of the study population was 71 years (range, 29 to 97 years), and $54 \%$ were men. LVEF measurement frequency and treatment decisions are illustrated in Figure 1. LVEF was measured before treatment in 128 patients $(65 \%)$. The modalities used to measure LVEF were two-dimensional echocardiography (77\%), MUGA scan (20\%), and cardiac magnetic resonance imaging (3\%). LVEF values were within normal range in 121 patients (95\%), and seven patients (5\%) had abnormal LVEF values. Three of these seven patients had prior clinical CHF (LVEF before $\mathrm{ABC}, 41 \%$ to $47 \%$ ), and the remaining four patients had incidentally detected ALVD (LVEF, $41 \%$ to $48 \%)$. Of the patients with normal LVEF values, 117 (97\%) were treated with $\mathrm{ABC}$; the remaining four (4\%) were not treated with $A B C$ per physician discretion because of advanced age (92 and 96 years), severe aortic stenosis, and poor performance status. Of the seven patients with abnormal LVEF, five were treated with $A B C$, including all four patients with ALVD; two patients had prior clinical $\mathrm{CHF}$ and did not receive $\mathrm{ABC}$. Of the 154 patients who received $\mathrm{ABC}, 153$ were administered the standard CHOP or R-CHOP regimen. The remaining patient had a history of CHF, had a current LVEF of $41 \%$, and was treated with an infusional doxorubicin regimen. Fifteen patients who had LVEF measured had been diagnosed with clinical CHF several years before their DLBCL diagnosis. In 12 of these patients $(80 \%)$, the results of the repeat LVEF measurement were within normal range, whereas the remaining three patients (20\%) had persistently low LVEF values (range, $41 \%$ to $47 \%)$.

LVEF was not measured in 69 patients (35\%; Fig 1). Of these, 32 were deemed by their attending physicians to be at clinically low risk for ALVD, and all received ABC. LVEF was not measured in the remaining 37 patients, because $A B C$ was not planned for the following reasons: medical frailty $(n=15)$, palliative care $(n=3), A B C$ not standard treatment $(n=12$; cutaneous or CNS lymphoma), and history of CHF $(n=7)$.

Among the group of 145 patients for whom $\mathrm{ABC}$ was intended or contemplated and who did not have a history of $\mathrm{CHF}$, the clinical features of those who did $(\mathrm{n}=113)$ and did not $(\mathrm{n}=32)$ undergo LVEF measurement are listed in Table 1 . Patients who did not have LVEF measured were likely to be younger (median age, $49 v 72$ years; $P<.001$ ) and to have fewer CHF risk factors (median, $1.5 v 2.0 ; P=.004$ ). At the time of this study, 88 patients (61\%) were still alive and had a median follow-up of 60 months (range, 8 to 138 months). Six (4\%) of the 145 patients were lost to follow-up. The rate of clinical CHF was not statistically different between those who did and did not have LVEF measured (16\% v 6\%, respectively; $P=.246)$. In the subgroup of patients who had LVEF measured, the test result did not affect treatment strategy (Fig 2). The median number of CHF risk factors was not statistically different between those who did and did not develop CHF (three $v$ two, respectively; $P=.474$ ).

We also reviewed the practice pattern among physicians for the rate of LVEF measurement among patients for whom $A B C$ was intended $(n=145)$. Because of the small number of patients with DLBCL seen, all oncologists were grouped together and compared with hematologists individually. Overall, more than half of the patients seen by each physician had LVEF measured, with the rates per physician varying significantly from $58 \%$ to $96 \%(P=.002)$. However, the rates of $\mathrm{CHF}$ after ABC per physician were not statistically different, regardless of LVEF measurement rates (data not shown). Of note, 19 patients were enrolled onto clinical trials, all of which required LVEF measurement as an entry criterion. 


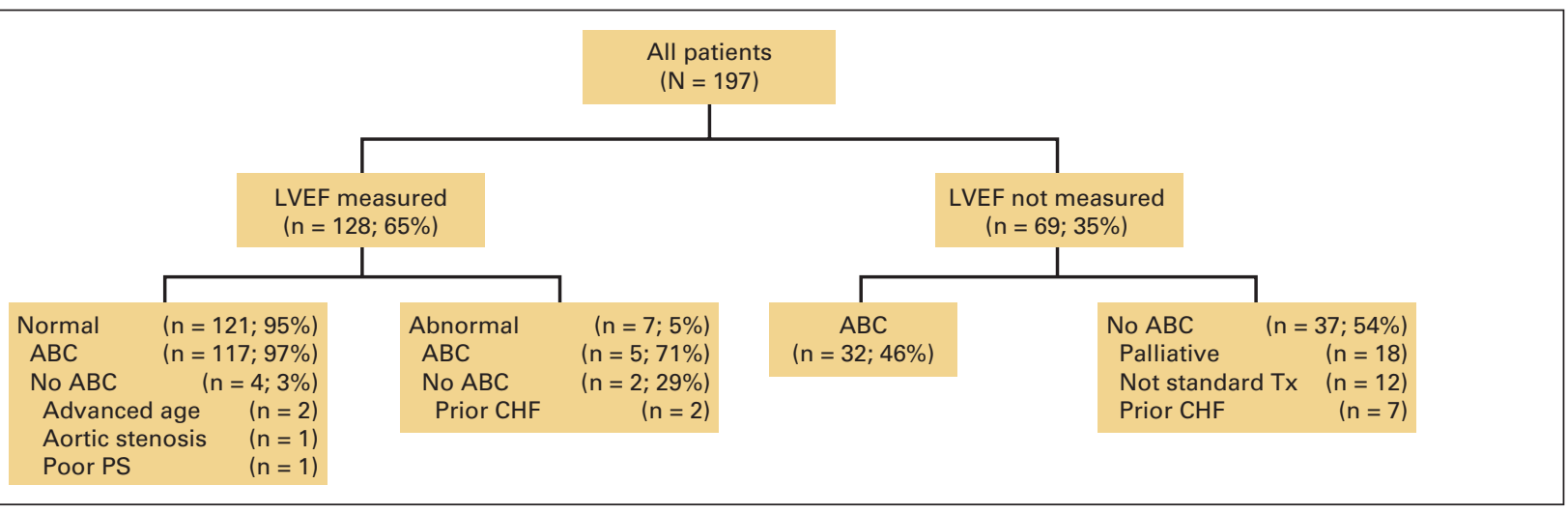

Figure 1. Flow diagram of all patients in the study. ABC, anthracycline-based chemotherapy; CHF, congestive heart failure; LVEF, left ventricular ejection fraction; PS, performance status, Tx, treatment.

Table 1. Demographic and Clinical Characteristics of Patients Without History of CHF Who Received ABC, by LVEF Measurement Status

\begin{tabular}{|c|c|c|c|c|c|}
\hline \multirow[b]{2}{*}{ Characteristic } & \multicolumn{2}{|c|}{ Measured } & \multicolumn{2}{|c|}{ Not Measured } & \multirow[b]{2}{*}{$P$} \\
\hline & No. & (\%) & No. & (\%) & \\
\hline Total No. of patients & 113 & & 32 & & \\
\hline Median age, years & 69 & & 51 & & $<.001$ \\
\hline Coronary heart disease & & 18 & & 6 & .162 \\
\hline Current smoker & & 32 & & 19 & .149 \\
\hline Diabetes mellitus & & 23 & & 3 & .009 \\
\hline Hypertension & & 59 & & 25 & .001 \\
\hline Male sex & & 50 & & 56 & .562 \\
\hline Obesity & & 40 & & 34 & .576 \\
\hline Chest irradiation & & 0.9 & & 0 & $>.999$ \\
\hline Prior anthracycline & & 0.8 & & 0 & $>.999$ \\
\hline Median No. of risk factors & 2 & & 1.5 & & .004 \\
\hline $\mathrm{CHF}$ after $\mathrm{ABC}$ & & 16 & & 6 & .246 \\
\hline
\end{tabular}

Abbreviations: ABC, anthracycline-based chemotherapy; CHF, congestive heart failure; LVEF, left ventricular ejection fraction.

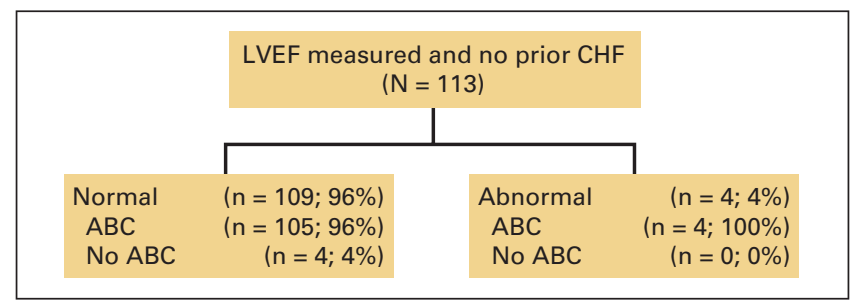

Figure 2. Treatment decisions after left ventricular ejection fraction (LVEF) measurement among patients without history of congestive heart failure (CHF). ABC, anthracycline-based chemotherapy.

\section{Discussion}

To our knowledge, this is the first published study investigating the utility of routine $L V E F$ measurement before $A B C$ in a large series of unselected patients with DLBCL. At our institution, this practice is common but not uniformly adopted. Among patients without a history of CHF, routine LVEF measurement did, on rare occasions, detect mild ALVD, but this finding did not affect the treatment decision. After several years of follow- up, post-ABC CHF rates were similar whether LVEF was measured before $\mathrm{ABC}$ or not. Our findings challenge the utility of routine LVEF measurement in patients with DLBCL before $\mathrm{ABC}$ as recommended by various US medical subspecialty clinical practice guidelines and as required in most US cancer cooperative group clinical trials. 5,6

For most patients, the rationale for measuring LVEF before $\mathrm{ABC}$ is to screen for ALVD. Although this makes intuitive sense, there are no data supporting this practice. On the contrary, at least three retrospective studies have shown the relative futility of this routine screening process. The first report by Dreicer et al, ${ }^{12}$ published in 1997, studied 222 patients seen at the University of Iowa who were considered for $\mathrm{ABC}$ in the treatment of hematologic $(34 \%)$ or solid $(66 \%)$ malignancies. Most (85\%) had LVEF measured by MUGA scan before, or at some point during, ABC. ALVD was detected in four patients (2\%) with LVEFs of $35 \%, 41 \%, 43 \%$, and $48 \%$, and $A B C$ was not administered. The investigators retrospectively commented that these four patients had LVEF values above the widely cited safe pretreatment value of $30 \%$ recommended by Schwartz et $\mathrm{al}^{9}$ and were therefore denied the potential benefit of ABC. A second study by Sabel et al, ${ }^{13}$ published in 2001, reviewed the practice of pretreatment MUGA scan in 98 women with breast cancer who were considered for adjuvant $\mathrm{ABC}$ at the Roswell Park Cancer Institute. No patient had prior heart disease. MUGA scan was performed in $62 \%$ of the patients before ABC. ALVD was found in two patients (2\%), each of whom had LVEF measured at $49 \%$. All patients went on to receive ABC. The third and most recent study was reported by Jeyakumar et $\mathrm{al}^{14}$ in 2012. This was a retrospective population-based study of 238 patients with stage I, II, or III breast cancer who received adjuvant chemotherapy. A majority of the patients (94\%) received ABC. MUGA scan was performed in 198 patients (83\%). Abnormal LVEF was observed in four (2\%; LVEF of $35 \%, 46 \%, 47 \%$, and $47 \%$ ), three of whom did not receive ABC. In all three studies, the study populations were relatively young (median or mean age, 47 to 58 years), predominantly composed of patients with breast cancer and patients at low risk for heart disease. Our study showed similar findings but in a much older population (median age, 71 years) 
with more risk factors for CHF and exclusively in patients with DLBCL.

In the setting of DLBCL, there are several reasons for not routinely measuring LVEF before ABC. First, the maximum cumulative dose of doxorubicin delivered when a full course of $\mathrm{R}-\mathrm{CHOP}$ is administered is relatively low-generally between 150 and $300 \mathrm{mg} / \mathrm{m}^{2}$ for patients with early- and advanced-stage DLBCL, respectively. This cumulative dose is much lower than the general recommendation of a maximum dose of $550 \mathrm{mg} /$ $\mathrm{m}^{2} .{ }^{15}$ Second, R-CHOP is a highly curative treatment for DLBCL regardless of stage and is the current standard of care. Even in the elderly population, 5- and 10-year progression-free survival rates are approximately $50 \%$ and $40 \%$, respectively. ${ }^{15}$ Finally, the risk of death resulting from $\mathrm{CHF}$ after R-CHOP among those who are most likely to be cured is relatively low: $4 \%$ at 5 years among those who had complete remission. ${ }^{16}$ Therefore, the benefits of $\mathrm{ABC}$ generally outweigh the risk of developing CHF in patients with no history of CHF. Furthermore, in our study, the rates of $\mathrm{CHF}$ after $\mathrm{ABC}$ among those did $(16 \%)$ or did not $(6 \%)$ undergo LVEF measurement were not statistically different. This may imply that history taking and physical examination are sufficient to screen patients for suitability of $A B C$, and $L V E F$ within normal range is not a good predictor of lower likelihood of CHF after ABC. However, a baseline LVEF measurement before $A B C$ is useful for some patients-for example, patients with prior CHF and patients for whom serial LVEF measurement is planned for clinical reasons.

We encourage the medical subspecialty groups responsible for developing relevant clinical practice guidelines and cancer cooperative groups to reevaluate the recommendation that LVEF be routinely measured before ABC. NCCN guidelines in particular are used extensively in oncology practices around the world and are being incorporated more frequently into practice quality measures, such as the Quality Oncology Practice Initiative. ${ }^{16,17}$ Although guidelines are neither designed to be followed in toto nor intended to represent a form of legal document, there are frequently legal concerns on the part of medical providers when care deviates from an established guideline. ${ }^{18}$ This is especially true when there is no clear-cut reason for not doing a test. For example, it is difficult to find a medical contraindication for measuring LVEF; on the other hand, clinical justifications for not administering $A B C$, no matter how subjective, are easily found. Similarly, cancer cooperative groups and academic cancer centers should reassess such a requirement in clinical trials. Most hematology-oncology fellows train in academic centers, and clinical trial enrollment is almost a routine treatment option. Practices or habits learned during the fellowship frequently become the standard of care after graduation.

In terms of health care costs, the savings from not routinely measuring LVEF before $\mathrm{ABC}$ can be substantial. In the United States alone, approximately 21,000 patients with newly diagnosed DLBCL are potentially eligible for $A B C$ each year. ${ }^{19}$ Current Centers for Medicare and Medicaid Services reimbursements for MUGA scan and limited two- dimensional echocardiography are approximately $\$ 332$ and $\$ 138$, respectively. ${ }^{20}$ Even if only half of these patients were routinely tested for LVEF, the cost of LVEF measurement alone could be between $\$ 2$ and $\$ 4$ million annually. If we extrapolate our findings to some of the other cancers in which $\mathrm{ABC}$ regimens are considered standard of care or an option-for example, acute leukemia (19,000 cases annually), stages II, II, and IV breast cancer (100,000 cases), Hodgkin lymphoma (8,000 cases), non-Hodgkin lymphoma other than DLBCL $(45,000$ cases $)$, and sarcoma $(11,000$ cases $)$ - the total cost savings is potentially much larger ( $\$ 14$ to $\$ 34$ million annually). ${ }^{19}$ These are extremely conservative estimates, because they are calculated using cost to the Center for Medicare and Medicaid Services rather than using charges or actual payments, which are usually several times higher.

Certain limitations apply to our findings. First, ours is a single-institution study of a moderate sample size. However, we included all patients seen at our institution with long-term and near-complete follow-up. Therefore, selection bias is minimized. Moreover, our results are consistent with the previous findings from other groups that predominantly studied patients with breast cancer. ${ }^{12-14}$ Second, although LVEF measurements were obtained in approximately $80 \%$ of the patients in our study for whom $\mathrm{ABC}$ was intended, the actual pattern of testing in community practice is unknown. To our knowledge, there is only one published practice pattern study, the findings of which suggest that community hematologists and oncologists obtain LVEF measurement in their patients before or during $A B C$ approximately $50 \%$ of the time. ${ }^{12}$ However, this was a retrospective survey of community physicians conducted 15 years ago and not based on actual medical record review. It is likely that the actual proportion of testing today is higher, because it is now recommended by major clinical practice guidelines. Furthermore, in the three published studies of LVEF measurement before $\mathrm{ABC}$, the actual institutional rates were $62 \%, 83 \%$, and $85 \% . .^{12-14}$ Finally, some of the LVEF measurements $(\mathrm{n}=19)$ were performed in the context of clinical trial participation and may not have been obtained if the patients were treated off study.

In conclusion, routine LVEF measurement before $A B C$ in patients with DLBCL with no history of CHF is a common, although not universal, practice at our institution. Mild ALVD was detected rarely, but presence of the condition did not affect the treatment decision. Our findings provide evidence contrary to current clinical practice guidelines and clinical trial requirements regarding the utility of routine LVEF measurement in those with DLBCL before ABC. Our study takes up the challenge set forth by the American Board of Internal Medicine Choosing Wisely campaign and the American Society of Clinical Oncology Cost of Care Task Force to question clinical practices that are not evidence based to provide more valuable care for our patients. ${ }^{21,22}$

Accepted for publication on July 19, 2012. 


\section{Acknowledgment}

Supported by the Gundersen Lutheran Center for Cancer and Blood Disorders and the Gundersen Lutheran Medical Foundation. Presented at the 46th Annual Meeting of the American Society of Clinical Oncology, June 4-8, 2010, Chicago, IL.

\section{Authors' Disclosures of Potential Conflicts of Interest}

The author(s) indicated no potential conflicts of interest.

\section{Author Contributions}

Conception and design: Amber L. Conrad, Vicki L. McHugh, Ronald S. Go

Administrative support: Ronald S. Go

Provision of study materials or patients: Ronald S. Go

\section{References}

1. Fisher RI, Gaynor ER, Dahlberg S, et al: Comparison of a standard regimen (CHOP) with three intensive chemotherapy regimens for advanced non-Hodgkin's lymphoma. N Engl J Med 328:1002-1006, 1993

2. Coiffier B, Lepage E, Briere J, et al: CHOP chemotherapy plus rituximab compared with $\mathrm{CHOP}$ alone in elderly patients with diffuse large-B-cell lymphoma. N Engl J Med 346:235-242, 2002

3. Habermann TM, Weller EA, Morrison VA, et al: Rituximab-CHOP versus $\mathrm{CHOP}$ alone or with maintenance rituximab in older patients with diffuse large B-cell lymphoma. J Clin Oncol 24:3121-3127, 2006

4. Pfreundschuh M, Trümper L, Osterborg A, et al: CHOP-like chemotherapy plus rituximab versus CHOP-like chemotherapy alone in young patients with good-prognosis diffuse large-B-cell lymphoma: A randomised controlled trial by the MabThera international trial (MInT) group. Lancet Oncol 7:379-391, 2006

5. National Comprehensive Cancer Network Clinical Practice Guidelines in Oncology: Non-Hodgkin's Lymphomas, Version 4.2011. http://www.nccn.org/ index.asp

6. Klocke FJ, Baird MG, Lorell BH, et al: ACC/AHA/ASNC Guidelines for the Clinical Use of Cardiac Radionuclide Imaging-executive summary: A report of the American College of Cardiology/American Heart Association Task Force on Practice Guidelines (ACC/AHAVASNC Committee to Revise the 1995 Guidelines for the Clinical Use of Cardiac Radionuclide Imaging). Circulation 108:1404-1418, 2003

7. Bedford Laboratories: Adriamycin. http://www.bedfordlabs.com/our_products/ online_catalog/products/doxorubicin.html

8. Von Hoff DD, Layard MW, Basa P, et al: Risk factors for doxorubicin-induced congestive heart failure. Ann Intern Med 91:710-717, 1979

9. Schwartz RG, McKenzie WB, Alexander J, et al: Congestive heart failure and left ventricular dysfunction complicating doxorubicin therapy: Seven-year experience using serial radionuclide angiocardiography. Am J Med 82:1109-1118, 1987
Collection and assembly of data: Amber L. Conrad, Jacob D. Gundrum, Ronald S. Go

Data analysis and interpretation: All authors

Manuscript writing: All authors

Final approval of manuscript: All authors

Corresponding author: Ronald S. Go, MD, FACP, Mail Stop: EB2001, Center for Cancer and Blood Disorders, Gundersen Lutheran Health System, 1900 South Ave, La Crosse, WI 54601; e-mail: rsgo@gundluth.org.

DOI: 10.1200/JOP.2012.000682; published online ahead of print at jop.ascopubs.org on September 18, 2012.

10. Swain SM, Whaley FS, Ewer MS: Congestive heart failure in patients treated with doxorubicin: A retrospective analysis of three trials. Cancer 97:2869-2879, 2003

11. He J, Ogden LG, Bazzano LA, et al: Risk factors for congestive heart failure in US men and women: NHANES I epidemiologic follow-up study. Arch Intern Med 161:996-1002, 2001

12. Dreicer R, Karwal MW, Midence G, et al: The role of radionuclide angiocardiography in the treatment of patients receiving doxorubicin-based chemotherapy: A reassessment. Am J Clin Oncol 20:132-137, 1997

13. Sabel MS, Levine EG, Hurd T, et al: Is MUGA scan necessary in patients with low-risk breast cancer before doxorubicin-based adjuvant therapy? Multiple gated acquisition. Am J Clin Oncol 24:425-428, 2001

14. Jeyakumar A, DiPenta J, Snow S, et al: Routine cardiac evaluation in patients with early-stage breast cancer receiving adjuvant chemotherapy. Clin Breast Cancer 12:4-9, 2012

15. Shan K, Lincoff AM, Young JB: Anthracycline-induced Cardiotoxicity. Ann Intern Med 125:47-58, 1996

16. Carlson RW: Industry "rewrites" of NCCN guidelines. J Natl Compr Canc Netw 9:257, 2011

17. The Quality Oncology Practice Initiative: Review of methodology and measures. http://qopi.asco.org/Methodology

18. Miller FH: The legal ramifications of the NCCN practice guidelines. Oncology (Williston Park) 10:35-39, 1996

19. Surveillance, Epidemiology, and End Results. http://seer.cancer.gov/

20. Centers for Medicare and Medicaid Services: Physician fee schedule. http:// www.cms.gov/apps/physician-fee-schedule/

21. Choosing Wisely: An initiative of the ABIM Foundation. http://choosingwisely. org/

22. Schnipper LE, Smith TJ, Raghavan D, et al: American Society of Clinical Oncology identifies five key opportunities to improve care and reduce costs: The top five list for oncology. J Clin Oncol 30:1715-1724, 2012 